$\frac{12}{6} / 26.95850$

UCRL-ID-119334

\title{
LIGHT EMITTING DIODE (LED) FIDUCIAL SYSTEM Setup and Operation
}

Steven A. Muelder

January 1995

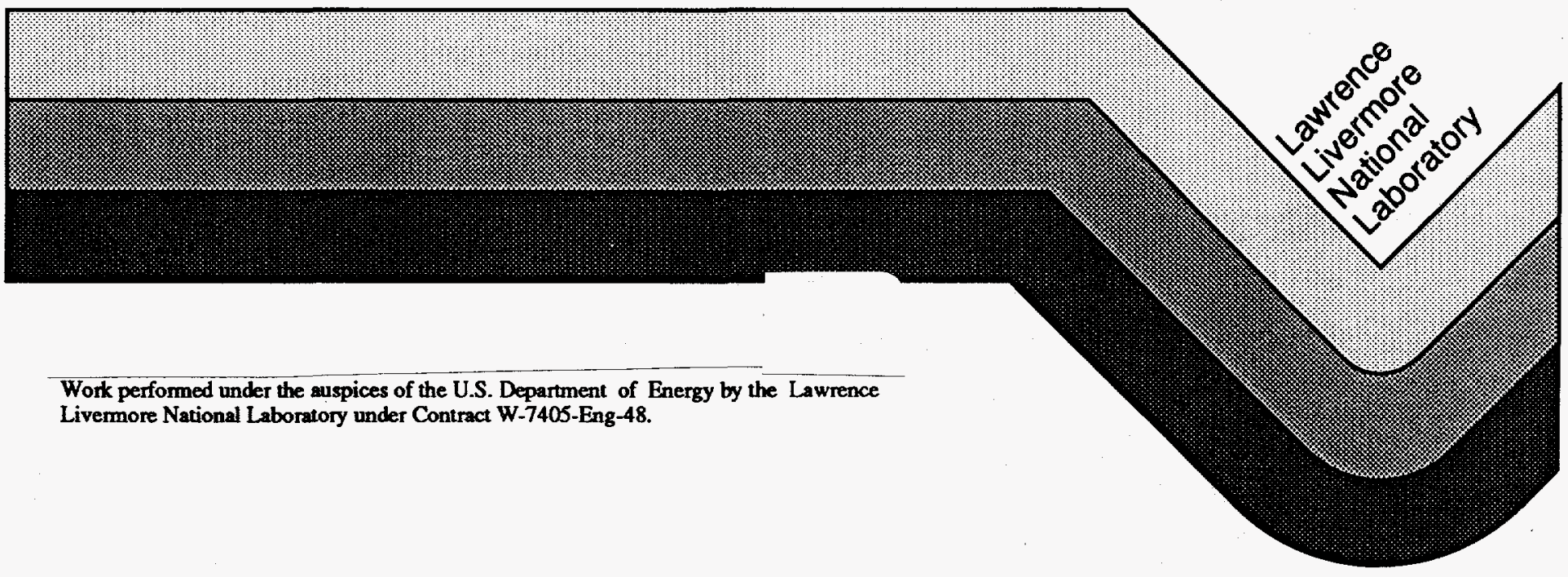




\section{DISCLAIMER}

This document was prepared as an account of work sponsored by an agency of the United States Government. Neither the United States Government nor the University of California nor any of their employees, makes any warranty, express or implied, or assumes any legal liability or responsibility for the accuracy, completeness, or usefulness of any information, apparatus, product, or process disclosed, or represents that its use would not infringe privately own rights. Reference herein to any specific commercial products, process, or service by trade name, trademark, manufacturer, or otherwise, does not necessarily constitute or imply its endorsement, recommendation, or favoring by the United States Government or the University of California. The views and opinions of authors expressed herein do not necessarily state or reflect those of the United States Government or the University of California, and shall not be used for advertising or product endorsement purposes.

This report has been reproduced directly from the best available copy.

Available to DOE and DOE contractors from the Office of Scientific and Technical Information

P.O. Box 62, Oak Ridge, TN 37831

Prices available from (615) 576-8401, FTS 626-8401

Available to the public from the

National Technical Information Service

U.S. Department of Commerce

5285 Port Royal Road

Springfield, VA 22161 


\section{DISCLAIMER}

Portions of this document may be illegible in electronic image products. Images are produced from the best available original document. 


\section{LIGHT EMITTING DIODE (LED) FIDUCIAL SYSTEM Setup and Operation \\ Steven A. Muelder, EE/DSED}

\section{INTRODUCTION}

Streak cameras, either electronic or rotating mirror, are common diagnostic tools used to explore very fast phenomena. Often they are used to precisely time events or durations, and in most cases, it is important to have quality time marks on the film record. Many methods have been used to introduce time marks onto the film. Exploding bridge wires are frequently used, but they light up slowly and are difficult to read with consistent accuracy. It is also difficult to put more than a few bridge wires in the view of a camera slit, so there are only a few timing marks written to the film. In some cases the time scale on the film must be interpolated over long distances, creating a significant loss of accuracy. Interpolation is especially troublesome with those cameras that have a nonlinear sweep rate. Spark gaps located on the camera slit turn on a bit faster, but suffer from the same interpolation induced errors. A series of short duration laser pulses, accurately timed and introduced along the edges of the film, is a very fine solution. The problem with such a laser fiducial system is that the lasers are very expensive and difficult to maintain. There has been a need for many years for an inexpensive, very bright light source that can be repetitively pulsed accurately and with short duration. Various technologies have sufficiently developed over the past few years, allowing us to build a fiducial system based on light emitting diodes (LED) for the Cordin rotating mirror streak cameras.

\section{BACKGROUND}

In order to understand the LED Fiducial System for the Cordin rotating mirror streaking cameras, it is appropriate to first review a bit of history. Many years ago, during the development of the electronic streaking cameras as used in the Fabry Perot velocimeters, Tony Huen devised a pulse burst generator that flashes LED's directly with very short pulses ( 30 to $40 \mathrm{nsec}$ ). The chassis that Tony built (commonly called the Tony box) has a keypad on the front from which the operator can input the number of pulses in the pulse burst, the pulse spacing in microseconds, and the positions of three fiducials within the burst. These fiducials are lower amplitude pulses, which cause identifiably dimmer light dots on the film record. In order to differentiate the three fiducials, the first is one low-amplitude dot, the second is two consecutive dim dots, and the third is three consecutive dim dots. The Tony box outputs pulses of nominally $12 \mathrm{~V}$ amplitude. This is sufficient to drive the LED's directly, since the LED's are mounted in the slit planes of electronic cameras that have image intensifiers on their outputs. The LED's are not bright enough to write directly to film, as would be necessary in using this technique with a mechanical camera that does not have an intensifier. Even as the LED's got brighter over the years, there still wasn't enough light. We still needed a way to get more light to the film. 
With the advent of high voltage, high power MOSFET's, it became possible to generate pulses of several hundred volts with risetimes of a few nanoseconds. This, married with the newest generation of superbright LED's (especially the shorter wavelength LED's which more closely matched the sensitivity of current films like T-Max), spawned a chassis called the LED Fiducial Driver (sometimes called the Rivera box after Tony Rivera, a principal in its development). The fid driver is essentially a waveshaper - when triggered by a 5 to 10 volt pulse, it puts out a -220 volt pulse of duration $18 \mathrm{nsec}$. This is about 100 times the normal drive voltage for the LED's, but it is a short enough duration that there is no evident damage to the LED's. There is, however, enough light to write to film directly. This then forms the basis for the LED Fiducial System, made up of a Tony box (to generate the burst of pulses with the desired characteristics), the fid driver (to transform the Tony box pulses to the high voltage pulses needed for the LED's), and camera slits with LED's embedded at the slit plane.

One difficulty with the fid driver is that it is triggered - it is not an amplifier. This means that it cannot discriminate against a Tony box pulse that is lower in amplitude. Either the fid driver is triggered or it is not. The Tony boxes used in the LED Fiducial System have to be modified to eliminate the three fiducials so they will not trigger the fid driver. These three fiducials in modified Tony boxes evidence themselves by being missing, not by having lower amplitude. A few years ago Jim Lawson put the heart of the Tony box in a CAMAC module. The so-called Lawson module does not have the external controls of the Tony box. It must be programmed remotely via the CAMAC highway. As with the Tony boxes, the Lawson modules must be modified for use with the LED Fiducial System for rotating mirror cameras. The Lawson modules are programmed at Bunkers 801 and 851 with a code called LEDFIDTT (written by Gary Turner). More about the programming of the modules later.

\section{LAWSON MODULE/TONY BOX}

The Lawson modules (and the Tony boxes) have a trigger input with TTL sensitivity. We've been told to be careful not to drive the inputs with more than 10-12 volts, so a typical trigger is limited to 5-8 volts. A $75 \mathrm{~V}$ det shaper pulse (with suitable attenuation) makes a fine trigger, since in most experiments it is a well defined time (and usually the most important time). Both the Lawson module and Tony box have three outputs - Burst A, Burst B, and Report. The two burst ports are identical - they put out $12 \mathrm{~V}$ pulse bursts at the same time. The report output puts out the missing pulses that are the fiducials of the burst outputs. Basically, the one, two, and three missing pulse fiducials go to the report output rather than the burst output. These are used to time where the fiducials are in relation to the trigger pulse (supposedly a known reference time like det shaper). The Lawson module (Tony box) has its first pulse out not at zero (which is not possible anyway), but after one interpulse period. The module in Bunker 801 (and perhaps the others as well) seem to have an additional delay of 25-30 ns. What this means is, if the module is setup to have 1.0 usec between 
pulses, the first pulse will be at about 1.025 usec after trigger and each subsequent pulse at 1.00 usec after the previous (i.e., at 2.025, 3.025, etc.).

\section{FIDUCIAL DRIVER}

The fid driver is connected to the burst output of the Lawson module or the Tony box. The fid driver is supposed to have a TTL input, but it is fairly sensitive and can be triggered by as little as $0.8 \mathrm{~V}$ (although the throughput delay increases as the trigger amplitude decreases). In order to be consistent, it is best to trigger the fid driver with 4-8 $\mathrm{V}$ pulses. That way the throughput delay of the fid driver stays at about $25 \mathrm{nsec}$ (it can go to as high as $40 \mathrm{nsec}$ for a $1 \mathrm{~V}$ trigger). To accomplish this, put a $2 X$ attenuator on the input to the fid driver if only one is being driven by a Lawson module. To trigger two fid drivers, use a 3-port splitter on the burst output of the Lawson module (the other burst output has another job, described below). Two of the splitter ports drive the two fid drivers with no attenuators (the splitter is a $2 X$ attenuator). To drive three fid drivers, put a second 3-port splitter on the other burst output of the Lawson module. If four or more fid drivers are needed, use a 5, 6, or 8-port splitter; the delay through the fid driver will have to be measured for each camera (or record the actual output pulses from each fid driver as discussed below). In order to protect the LED's in the camera slit, the fid driver has limitations built into its circuitry. The maximum number of pulses without a drop in pulse amplitude is 100 and the maximum pulse rate is $2.5 \mathrm{MHz}$ ( 0.4 usec between pulses).

\section{TIMING MEASUREMENTS}

The fid drivers have a monitor output that samples the output voltage pulses to the LED's in the slit. These signals can be recorded on transient digitizers (like the LeCroy TR8828), on digital storage oscilloscopes (like a Tektronix 544A), or on scopes equipped with cameras. This is the best way to confirm that the diodes have actually been pulsed and the precise times of the pulses (as long as all the cable lengths are known and the trigger signal is also recorded). The CAMAC crate containing the Lawson module also contains a LeCroy 4208 TDC (Time-toDigital Converter). The same trigger (det shaper) that triggers the Lawson module should trigger the TDC. The unused burst signal from the Lawson module is connected to the TDC Port 5 (attenuated to about 3-5 volts). This signal is used to measure the pulse-to-pulse spacing. The report output of the Lawson module is connected via a $5 \mathrm{X}$ attenuator to the port 1 of the TDC. The TDC is configured to measure and report the time from trigger to the time of the first of each report pulse (so it measures the time from trigger to the first missing pulse in each of the three fiducials of missing light spots). This then forms an absolute time reference for the film record to a supposedly known time (the trigger signal).

\section{SETTING PARAMETERS}

The pulse burst parameters of the Lawson module must be set via the bunker computer system. In Bunker 801, log onto the bunker system from the cursor 
SVR801 > by typing LOG [enter]. The user name is LED and the password is FID. Once on the SVR801, you must type RMT BMC801 [enter]. This allows access to the BMC from a remote terminal. Log onto the BMC the same way (type LOG [enter], followed by user LED and password FID). The program LEDFIDTT will start automatically. LEDFIDTT is menu driven by the first letter of the desired command. From the main menu, type F(ids) to set parameters, T(iming) to arm the TDC for measurement, and Q(uit) to end the program. The fid menu allows the $P$ (eriod) to be set in $x . x$ microseconds, the $R$ (ange) of the burst in microseconds, and the times of the 1(st), 2(nd), and 3(rd) fiducials (the missing pulses from the burst). The timing menu has I(nitialize) to arm the TDC; both menus use $M$ to revert to the main menu. Be aware that there have to be at least 5 pulses before the first fiducial blank or the TDC measurement of period will be inaccurate due to the way in which the TDC is internally configured to make the measurement. After the TDC and Lawson modules are triggered, LEDFIDTT queries the TDC and displays the information on the timing menu page. It will list the period between pulses and the times from the trigger to each of the three fiducial blanks (to the first missing pulse in each fiducial string). After quitting LEDFIDTT, type LOG OFF [return] (or simply LO) to log off the BMC and LO again to $\log$ off the SVR801.

It is always a good idea to manually pulse the Lawson module/Tony box anytime the parameters are changed, to assure that the digital registers in the circuits are updated. Next, pulse the system with the TDC armed to verify that the timing is correct. If there is time, repeated arming and firing of the system will net some statistics on jitter. The three fiducial markers sometimes tend to vary by 5 to $10 \mathrm{nsec}$, as measured by the TDC. Manual system firing can be done by substituting a "grey box" pulser for the det shaper signal. It also makes sense, if the equipment is available, to directly monitor the output of the LED drivers via the 50:1 voltage divider monitor port. The monitor output will be a burst of $-4.4 \mathrm{~V}$ pulses with missing pulses at the appropriate locations. Using a LeCroy 8828 or a Tektronix $544 \mathrm{~A}$, accurate measurements can be made of the entire pulse burst. The normal waveshapes are relatively clean, so pulse echoes or "hash" on the output are indicative of LED's that have failed or some other fault condition in the slit or drive cable.

\section{TIMING ANALYSIS}

A diagram of a "typical" Bunker 801 setup is attached. Assume that the Lawson module has been programmed with a $0.5 \mathrm{usec}$ period $(2.0 \mathrm{MHz})$ and markers at pulses 6,12 , and 20. This means that the pulses at about 3.0, 6.0 and 6.5 and $10.0,10.5$, and 11.0 usec should be missing from the film record. After the shot, the TDC readout lists the period at 0.500 usec and the markers at 3.025, 6.025, and 10.026 usec. The analysis of the actual fiducial times proceeds as follows (refer to the oneline diagram).

The triggers for the 4208 and Lawson modules arrive $-138+138+20+2+100+10+1$ $=133 \mathrm{nsec}$ after the det pulse arrives at the shot. The first pulse out of the Lawson 
module will be $500+25 \mathrm{nsec}$ after trigger arrival (the module has a $25 \mathrm{nsec}$ delay built in plus the delay from " 0 " to the first pulse, i.e., the period), so the first pulse arrives at the LED driver $133+525+1+50=709 \mathrm{nsec}$ after detonator initiation. The LED driver has an internal delay of $25 \mathrm{nsec}$, so the first pulse gets to the slit at $709+25+10=744 \mathrm{nsec}$. The pulses are $20 \mathrm{nsec}$ wide, so the center of the light pulse is at $754 \mathrm{nsec}$ after the shot is initiated. The first fiducial marker is pulse \#6, which is $5 * .500=2.50$ usec after pulse \#1, hence $2500+754=3254$ nsec after detonator start. To be absolutely precise, the distance from the shot to the slit plane must also be accounted for. Light travels about 1 foot per nsec (11.8 inches in vacuum). If, for example, we assume 30 feet from the shot to the camera, then the actual film record is delayed by $30 \mathrm{nsec}$. The first missing light pulse would then correspond to $3224 \mathrm{nsec}$ from det initiation (the way to view this is to realize that there is no difference between having a $138 \mathrm{~ns}$ det cable to the shot followed by 30 feet of air and $168 \mathrm{~ns}$ of det cable and the shot right at the camera - the overall delay is the same). Since all the cables going to the Tek 544A are $50 \mathrm{ns,}$ the scope measures the real time delay from trigger signal to driver output. Add to this measurement $10 \mathrm{~ns}$ for the cable to the slit and $10 \mathrm{~ns}$ to get from the edge to the center of the pulse. Actual measurements on three different shots are 610, 618 , and $614 \mathrm{~ns}$ for the times from trigger to the center of the first pulse. Estimated time is $1+500+$ approx $25+1+50+$ approx $25+10=612 \mathrm{nsec}$. The film can be read to a precision of about $\pm 10 \mathrm{nsec}$, so this is about as accurate as the system needs to be and certainly more precise than trying to read bridge wires. 
TYPICAL SETUP FOR LED FIDUCIAL SYSTEM BUNKER 801

BUNKER

TRIISGER

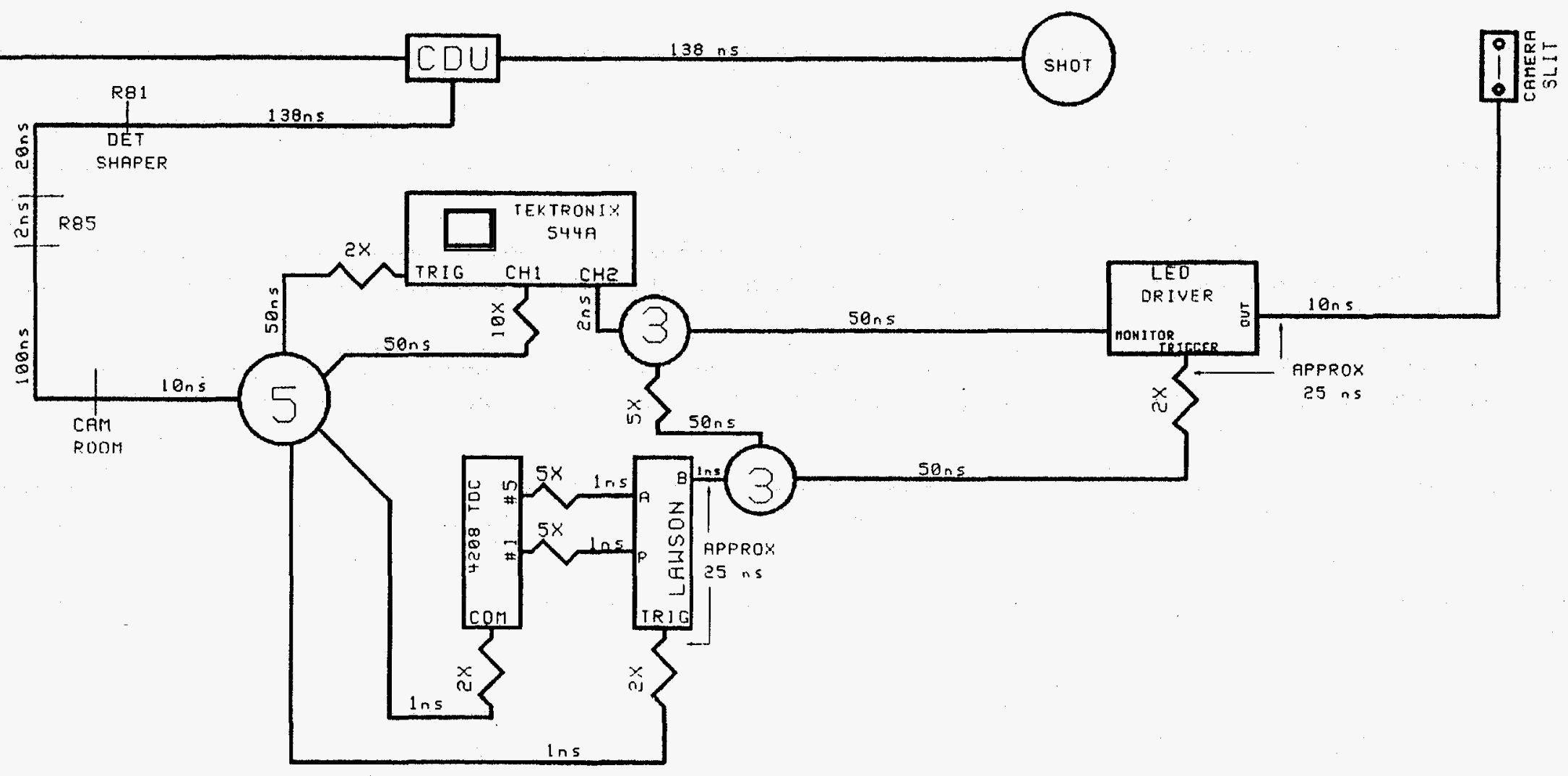

